\title{
A Numerical Method of Failure Process of Geotechnical Materials
}

\author{
Yanqiong LIU \\ China Institute of Engineering Mechanics, Key Laboratory \\ of Earthquake Engineering and Engineering Vibration, \\ China Earthquake Administration \\ Beijing, China \\ e-mail: Yanqiong-liu@163.com \\ Peixuan LIU \\ China National Earthquake Infrastructure Service \\ Beijing, China \\ e-mail: 1px@163.com
}

\begin{abstract}
In this paper, the displacement discontinuity initiation conditions are deduced based on potential energy principle. The finite element discrete of weak form of governing equation is developed by combination of displacement discontinuity evolution conditions and discontinuity connection which material derivative describes.
\end{abstract}

Keywords-potential energy principle; displacement discontinuity; discontinuity connection; discretization

\section{DESCRIPTION OF THE PROBLEM}

A solid analysis domain is V. Its stress boundary is $S_{\sigma}$ and displacement boundary is $S_{u}$. The equilibrium equation is

$$
\nabla \sigma+b=\rho \ddot{u}
$$

The constitutive relation is

$$
\sigma=D \varepsilon \text { or } \dot{\sigma}=D \dot{\varepsilon}
$$

When in the elastic stage, $D=D^{e}$. When in the elastic-plastic stage, $D=D^{e}-D^{p} . D^{p}$ is related to loading condition, plastic potential function and plastic stiffness of material. The strength conditions of solid failure are analyzed:

$$
\Phi\left(\sigma, \sigma_{c}(q)\right)=0 \quad \text { or } f(\sigma)-\sigma_{c}(q)=0
$$

It is our aim to establish numerical analysis method that satisfy the formulas (1) (3) and displacement discontinuity failure process of Initial boundary value condition, under the quasi-static loading condition(See Fig.1).The analysis process consists three main parts: discontinuous initiation and merisis, discontinuous connection and displacement discontinuity evolution.

\author{
Jisheng ZHAO \\ China National Earthquake Infrastructure Service \\ Beijing, China \\ e-mail: Jisheng_zhao@163.com
}

Hongsuai LIU

China National Earthquake Infrastructure Service Beijing, China e-mail: 13810892160@163.com

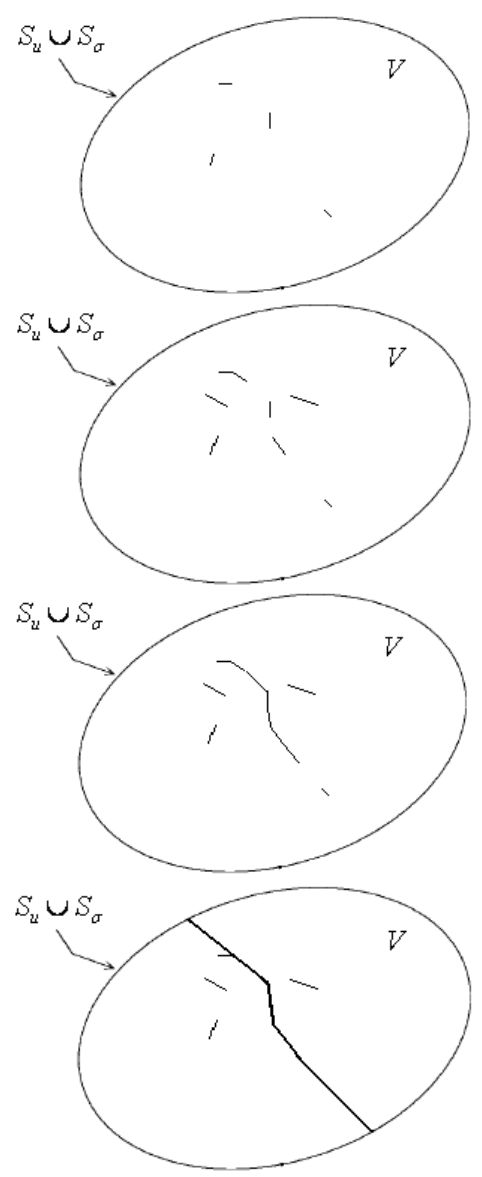

Figure 1. Failure process of solid

\section{THE DISPLACEMENT DISCONTINUITY CONDITIONS}

When quasi-static is loading, the internal displacement of the solid occurs. That is $u=\bar{u}+u H_{u}$. The force on both sides of the discontinuity surface can be regarded as an external force. 
The Corresponding potential energy principle is:

$$
\begin{aligned}
& \Pi=\int_{V \backslash j^{ \pm}} W d V-\int_{V} b u d V-\int_{S_{\sigma}} \phi u d S-\int_{S_{j^{+}}} \phi^{\prime} u^{+} d S-\int_{S_{j^{+}}} \phi^{\prime} \llbracket u^{+} \rrbracket d S \\
& -\int_{S_{j^{-}}} \phi^{\prime \prime} u^{-} d S-\int_{S_{j^{-}}} \phi^{\prime \prime} \llbracket u^{-} \rrbracket d S
\end{aligned}
$$

In this formula, $W=\int_{0}^{\varepsilon} \frac{\partial w}{\partial \varepsilon} d \varepsilon, w$ is strain energy density.

$\delta \Pi=0$, that is:

$$
\begin{aligned}
& -\int_{V \backslash j^{\delta}} \delta u(\nabla \sigma+b)+\int_{S_{\sigma}} \delta u(n \sigma-\phi)+\int_{s_{J_{+}}} \delta u^{+}\left(n^{+} \sigma-\phi^{\prime}\right)+\int_{s_{f^{+}}} \delta \llbracket u^{+} \rrbracket\left(n^{+} \sigma-\phi^{\prime}\right) \\
& +\int_{S_{j^{-}}} \delta u^{-}\left(n^{-} \sigma-\phi^{\prime \prime}\right)+\int_{S_{j^{-}}} \delta \llbracket u^{-} \rrbracket\left(n^{-} \sigma-\phi^{\prime \prime}\right)=0
\end{aligned}
$$

The left top three items of the formula (5) respectively correspond to equilibrium equation, stress boundary and stress coordination condition of the discontinuity surface. We are concerned about the six and fourth. They must conform to the strength condition: $\phi^{\prime}=n^{+} \sigma_{c}$ and $\phi^{\prime \prime}=n^{-} \sigma_{c}$. The displacement discontinuity condition is

$$
\delta \llbracket u^{-} \rrbracket\left(n^{-} \sigma-\phi^{\prime \prime}\right)=0
$$

There is no limit to stress and strain matrix, so the above conditions may be elastic matrix and elastic plastic matrix .Beyond that, strain hardening and strain softening are also considered.

When the failure strength $\sigma_{c}$ changes with $u$

or $\dot{u}$ (especially the decrease), quasi-static equilibrium state is broken and the analysis domain appears unbalanced force. The result is that the static equilibrium state is changed into the dynamic equilibrium state. Вy $\delta \Pi=0$, taking into account the formula (5) and the change of motion state of analyzed object. At a certain moment $t$,

$$
-\int_{S_{j^{+}}} \delta \phi^{\prime} \llbracket u^{+} \rrbracket-\int_{S_{j^{-}}} \delta\left(\phi^{\prime \prime}\right) \llbracket u^{-} \rrbracket=-\int_{V} \rho \delta u \cdot \ddot{u}
$$

When the displacement / velocity of the displacement of the discontinuity surface are weakened, the analysis domain will appear the phenomenon that displacement discontinuity accelerates the destruction. However, when the displacement / velocity of the interface is strengthened, the damage evolution is not obvious, unless the loading continues to strengthen.

When analysis domain is analyzed as a whole, through the relationship $\phi^{\prime}=n^{+} \sigma_{c}, \phi^{\prime \prime}=n^{-} \sigma_{c}, u^{+}-u^{-}=0$, $\llbracket u^{+} \rrbracket-\llbracket u^{-} \rrbracket=u$ and formula (4), it can be rewritten as:

$$
\Pi=\int_{V \backslash S_{j^{+}}} W d V-\int_{V \backslash S_{j^{+}}} f \bar{u} d V-\int_{S_{\sigma}} \phi \bar{u} d S+\int_{S_{j^{+}}} \phi^{\prime \prime} \quad u d S
$$

The last item of the formula (8) is the product of the force on one side of the displacement discontinuity interface and the slip distance of the other side. It shows the consumption of system energy. At the same time, this paper focuses on displacement discontinuity failure processes as the result of the failure strength $\sigma_{c}$ changes with

$u$.The formula can be concluded:

$$
\begin{gathered}
\Pi=\int_{V \backslash S_{j}} W d V-\int_{V \backslash S_{j}} b u d V-\int_{S_{\sigma}} \phi u d S+\int_{S_{j}} n^{-} \sigma_{c} u d S \\
\Lambda=\int_{V} \frac{1}{2} \rho \dot{u}^{2} d V
\end{gathered}
$$

In the above formulas, $\Lambda$ is the system function.

Taking into account the differences of the constitutive description of the different time stages and different locations in the analysis domain, and stress vector independent existence in weak form. Applying the formula Hamilton, the weak form $\delta \Pi-\delta \Lambda=0$ of the governing equation at the moment of $\mathrm{T}$ is obtained. Specifically,

$$
-\int_{V} \rho \delta u \cdot \ddot{d} d V=\int_{V \backslash S_{j}} \nabla \delta u: \sigma d V-\int_{V} \delta u \cdot b d V-\int_{S_{\sigma}} \delta u \cdot \phi d S+\int_{S_{j}} \delta u\left(n^{-} \sigma_{c}\right) d S
$$

\section{THE INITIATION AND EVOLUTION OF DisPlaCEMENT DISCONTINUITY LINE}

\section{A. The Detection of the Initiation Condition}

The failure of the material is due to the instability of the materialism, but not related to rate. (Legendre-Hadamard condition) However, the mechanism that from the instability of the material to the discontinuous displacement is not very clear, and there are a lot of controversies over the destruction of material instability [2 4]. We start with the failure strength of the material directly. The condition that the material is not damaged must satisfy the inequality of the description form of the material point below:

$$
E(x)=\Phi\left(\sigma, \sigma_{c}(q)\right)>0
$$

In the formula, $\Phi\left(\sigma, \sigma_{c}(q)\right)$ based on the stress of the damage condition. It may be the friction criterion or the tensile stress criterion. The failure mode is corresponding to the formula (6).

On the basis of the description of the formula (10), for a given time and a material point in a solid.

$$
e(x):=\min \left(\Phi\left(\sigma, \sigma_{c}(q)\right)\right)
$$

In the formula, e is called damage index. When a point 
indicator e is absolutely positive, the corresponding material point is in good condition.

Note: Indicator in different coordinates

Formula (11) is the indicator of the overall coordinate. The angle between the normal direction of the discontinuity surface and the whole coordinate is obtained. It is convenient that indicator uses local canonical coordinates, when the analysis of finite element numerical value in equal parameter style is used. By $\mathbf{x}(\xi)=N \cdot \mathbf{x}^{\text {node }}$, the angle between the normal direction of the discontinuity surface and the local coordinate can be got. Such as two-dimensional problems, when the normal equation is known, the $x(\xi, \eta)=N(\xi, \eta) \cdot x^{\text {node }}$ and $y(\xi, \eta)=N(\xi, \eta) \cdot y^{\text {node }}$ into $y(x)=k x$ or $y(x)=\tan \left(\alpha^{x}\right) x$, which can calculate the normal equation of local coordinates: $\eta(\xi)=k^{\xi}\left(N, x^{\text {node }}, y^{\text {node }}\right) \cdot \xi$.

\section{B. Discontinuous Evolution}

Due to the displacement discontinuity is made of the material points that the index e lost positive, at the moment of $\mathrm{t}$, discontinuous tip indices should meet:

$$
\begin{aligned}
& e\left(x^{t i p}(t), t\right)=0 \\
& \dot{e}\left(x^{t i p}(t), t\right)=0
\end{aligned}
$$

Using the formula (12), the position of the discontinuous failure point and the discontinuous direction can be obtained. From the formula (13a) we obtain:

$$
\dot{e}(x)=\frac{\partial e}{\partial t}+\left(\nabla e^{t i p}\right) \cdot \dot{x}^{t i p}=0
$$

In the formula, $\dot{x}^{\text {tip }}$ is speed vector of intermittent tip and can be expressed as $\dot{x}^{t i p}=v^{t i p} \mathbf{a}^{t i p}, \mathbf{a}^{\text {tip }}$ is unit vector in the direction of slip, $v^{\text {tip }}$ is the evolution speed of intermittent tip. According to the formula (6), $\mathbf{a}^{\text {tip }}$ is the tangential direction of slip surface in the case of sliding and $\mathbf{a}_{T}^{\text {tip }}$ is the normal direction of slip surface in the case of disconnection.

Put $\dot{x}^{\text {tip }}=v^{\text {tip }} \mathbf{a}^{\text {tip }}$ into the formula (13b) and the length of discontinuous surface is expressed as $l$ to obtain the formula (14).

$$
\frac{d l}{d t}=-\frac{1}{\nabla e^{t i p} \cdot \mathbf{a}^{t i p}}\left(\frac{\partial e^{t i p}}{\partial t}\right)
$$

Based on the formula, the evolution position of single intermittent tip is traced. Determining slip surface can come down to the determination of the unit vectors of normal direction and tangential direction of slip surface. The common failure modes of displacement discontinuity in solid include open crack and contact-sliding. Actually, in general failure case the unit vector in the direction of $\operatorname{slip} \mathbf{a}^{t i p}$ is free between normal direction and tangential direction.

\section{COSTITUTIVE RELATION}

\section{A. Solid Interior $\left(V \backslash S_{j}\right)$}

In the case of small strain and non-associated plasticity, elastic-plastic stress-strain relation is

$$
\begin{gathered}
C^{e p}=C-\frac{\left(C: m^{*}\right) \otimes(m: C)}{H+m^{*}: C: m} \\
\lambda=\frac{(m: C): \dot{\varepsilon}}{H+m^{*}: C: m}
\end{gathered}
$$

In the formula, $m^{*}=\frac{\partial \Xi(\sigma, q)}{\partial \sigma}, m(\sigma)=\frac{\partial \Sigma(\sigma, q)}{\partial \sigma}$, $H=-\frac{\partial \Sigma}{\partial q} \frac{\partial q}{\partial \varepsilon^{p}} \frac{\partial \Xi}{\partial \sigma}$.

$C$-The tensor of elastic modulus.

$\Sigma(\sigma, q)$-Yield function.

$\Xi(\sigma, q)$-Plastic potential function.

$q$-Internal variable.

\section{B. Interface Model ( $\left.S_{j}\right)$}

In the analysis of displacement discontinuity failure, interface is idealized as a geometric surface. With increase of loads material gradually deteriorates to form a strong deformation zone. It further develops into displacement discontinuity, which makes the stress of displacement discontinuity surface tend to infinity. The displacement and stress on displacement discontinuity surface are assumed to be the following form.

$$
\begin{gathered}
u(x, t)=\bar{u}(x, t)+H_{S_{j}} u(x, t) \\
\varepsilon(x, t)=\nabla^{s} \bar{u}+H_{S_{j}} \nabla^{s} u+\frac{1}{h}\left(\begin{array}{l}
u \otimes n)^{s}
\end{array}\right.
\end{gathered}
$$

In the formula, $H_{S_{j}} u(x, t)$ is a slop function which width is $h$. When $h=0$, slop function changes into step function, which manifest on displacement discontinuity. Magnified indefinitely the last item on the right of the formula (17) to obtain the formula (18).

$$
\dot{\sigma}=C^{e p}:\left[\nabla^{s} \dot{\bar{u}}+H_{S_{j}} \nabla^{s} \dot{u}+\frac{1}{h}(\dot{u} \otimes n)^{s}\right]
$$


The fist two items on the right of the formula (17) are assumed to be elastic strains. And the last one is assumed to be plastic strain. Based on elastic relation, flow rule and plastic consistency condition factorize the formula (18) to obtain the following ones.

$$
\begin{gathered}
\dot{\sigma}=C:\left[\nabla^{s} \dot{\bar{u}}+H_{S_{j}} \nabla^{s} \dot{u}\right] \\
\frac{1}{h}(\dot{u} \otimes n)^{s}=\lambda m^{*}
\end{gathered}
$$

It is assumed that $H=\bar{H} h$, then

$$
\frac{\partial \Sigma}{\partial q} \dot{q}=-h \bar{H} \lambda
$$

Put the formula (19c) into the formula (19b) then divide $(1 / h)$ to obtain

$$
(\dot{u} \otimes n)^{s}=-\frac{1}{\bar{H}} \frac{\partial \Sigma}{\partial q} \dot{q} m^{*}
$$

Based on consistency condition, then

$$
(\dot{u} \otimes n)^{s}=\frac{1}{\bar{H}}(m: \dot{\sigma}) m^{*}
$$

The constitutive models of discontinuous interface are the formula (19a) and (20a) or the formula (19a) and (20b). The formula (19a) is always neglected in analysis because plastic strains are much greater than elastic ones when displacement discontinuity happens. According to the formula (20b) and the conditions of stress continuous, the explicit relationship between slip distance and failure stress of slip surface is set up.

$$
\left(n^{-} \dot{\sigma}_{c}(t)\right)_{V \backslash S_{j}}=F(\dot{u})
$$

In the formula, according to different stress failure criterion and corresponding relation of elements different representation of $F(\circ)$ is obtained.

\section{ESTABLISHMENT OF DISCRETE EQUATION}

Unit $u_{t}$ function space satisfying Dirichlet condition on $S_{u}$ is represented as $\pi_{t}$. Appropriate test function space is

$$
\vartheta:=\left\{\delta u ; u_{t}+\delta u \in \pi_{t}, u_{t} \in \pi_{t}\right\}
$$

Approximate space of displacement field is

$$
\pi_{t}^{h}:=\left\{u_{t}^{h} \in \pi_{t} ; u_{t}^{h}=u^{h}(\cdot, t) \text { in } V,\left.u_{t}^{h}\right|_{S_{u}}=u^{*}(t)\right\}
$$

In the formula, $u^{*}(t)$ is specified displacement on $S_{u}$ at time t. Discrete test space of finite element corresponding with the formula (9) is defined as

$$
\vartheta^{h}:=\left\{\delta u^{h} \in \vartheta ; \delta u_{t}^{h}=u^{h}(\cdot, t) \text { in } V,\left.\delta u^{h}\right|_{s_{u}}=0\right\}
$$

On the basis of displacement field $u(x, t)$, speed field $\dot{u}(x, t)$ and acceleration field $\ddot{u}(x, t)$ at a given time $\mathrm{t}$, we solve the displacement field at the next moment $t+\Delta t$ which satisfies $u_{t+\Delta t} \in \pi_{t+\Delta t}$. Before there is no discontinuous point analysis field is standard weak form of the governing equation. After there is discontinuous point weak form of the governing equation in analysis field is the formula (9). Approximate displacement field and acceleration field are

$$
\begin{aligned}
& u^{h}(x, t)=\sum_{I=1}^{N_{\text {node }}} N_{I}(x) \delta_{I}(t)+\sum_{I \in J} \Upsilon_{I} q(t) \mathbf{a}(x) \\
& \ddot{u}^{h}(x, t)=\sum_{I=1}^{N_{\text {node }}} N_{I}(x) \ddot{\delta}_{I}(t)+\sum_{I \in J} \Upsilon_{I} \ddot{q}(t) \mathbf{a}(x)
\end{aligned}
$$

In the formula (25), $N_{\text {node }}$ is number of nodes. $N_{I}$ is standard finite element shape function related to node I. J is relevant set of reinforced nods. $\delta_{I}$ is displacement degree of freedom of standard finite elements. $\mathbf{a}(x)$ is unit vector in slip direction. $q_{I}$ is displacement degree of freedom associated with enhancement. $\Upsilon_{I}$ is enhancement function and can be defined as

$$
\Upsilon_{I}=N_{I}(x)\left[H\left(f(x)-H\left(f\left(x_{I}\right)\right)\right]\right.
$$

In the formula (26), $H(\circ)$ is step function through one position. When $\mathrm{x}$ is greater than $0, \mathrm{H}(\mathrm{x})=1$. And when $\mathrm{x} \leq 0$, $\mathrm{H}(\mathrm{x})=-1 . \quad x_{I}$ is position vector of node $\mathrm{I} . \quad f(x)$ is position function of internal slip surface $S_{j}$, which depends on damage index of material point.

\section{A. Spatial Discretization Schemes}

The formula (25) describes displacement potential function of the field containing displacement discontinuity. The weak form of the formula (9) is rewrote into

$$
-\int_{V} \rho \delta u^{h} \cdot \ddot{u}^{h} d V=\int_{V \backslash S_{j}} \nabla \delta u^{h}: \sigma d V-\int_{V} \delta u^{h} \cdot b d V-\int_{S_{\sigma}} \delta u^{h} \cdot \phi d S+\int_{S_{j}} \delta u\left(n^{-} \sigma_{c}\right) d S
$$


Put displacement approximation, acceleration approximation and test function into the formula (9) and obtain semi discrete weak form.

$$
\begin{aligned}
& \sum_{I=1}^{\text {Nnod }}\left(\delta \delta_{I}\right) \cdot\left(K_{I K}^{\delta \delta} \delta_{K}+K_{I K}^{\delta q} q_{K}+M_{I K}^{\delta \delta} \ddot{\delta}_{K}+M_{I K}^{\delta q} \ddot{q}_{K}-f_{I}^{e x t}\right) \\
& +\sum_{I \in J}\left(\delta q_{I}\right) \cdot\left(K_{I K}^{q q} q_{K}+K_{I K}^{q \delta} \delta_{K}+M_{I K}^{q q} \ddot{q}_{K}+M_{I K}^{q \delta} \ddot{\delta}_{K}-Q_{I}^{e x t}\right)=0
\end{aligned}
$$

In the formula (28),

$$
\begin{gathered}
K_{I K}^{\delta \delta}=\int_{V \backslash S_{j}} \nabla N_{I} C^{e p} \nabla N_{K} d V \\
K_{I K}^{\delta q}=\int_{V \backslash S_{j}} \nabla N_{I} C^{e p} \nabla\left(\Upsilon_{K} \mathbf{a}\right) d V \\
K_{I K}^{q q}=\int_{V \backslash S_{j}} \nabla\left(\Upsilon_{I} \mathbf{a}\right) C^{e p} \nabla\left(\Upsilon_{K} \mathbf{a}\right) d V \\
M_{I K}^{\delta \delta}=\int_{V \backslash S_{j}} N_{I} \rho N_{K} d V \\
M_{I K}^{\delta q}=\int_{V \backslash S_{j}} N_{I} \rho\left(\Upsilon_{K} \mathbf{a}\right) d V \\
M_{I K}^{q q}=\int_{V \backslash S_{j}} \Upsilon_{I} \rho \Upsilon_{K} d V \\
\Upsilon_{I}(\phi \mathbf{a}) d S+\int_{V \backslash S_{j}} \Upsilon_{I}(b \mathbf{a}) d V-\int_{S_{j}} \Upsilon_{I}\left(n^{-1} \sigma_{c}\right) \mathbf{a} d S \\
f_{I}^{e x t}=\int_{S_{\sigma}} N_{I} \phi d S+\int_{V \backslash S_{j}} N_{I} b d V
\end{gathered}
$$

\section{B. Time Discretization}

After semi discrete weak form of governing equation is obtained the method from the literature six is developed for the temporal discretization of it. The stability and accuracy of the discrete time step needs to be analyzed.

\section{Quadrature}

The integrand of the discretization of governing equations is non-continuous on $S_{j}$. And the direction of discontinuous evolution is arbitrary. Standard integration scheme is not well suitable for the units containing discontinuity. So Delaunay triangle is used to divide discontinuous element into two parts. It is should be noted that these triangles are only used for integration and don't increase the freedom. To sum up, there are continuous passing through, discontinuous passing through and partial passing through according to the difference of integration. The first two are in progress based on the formula (26). And to cope with the units of partial passing through setting up the expression of enhancing shape function is in demand.

\section{CONCLUSION}

In this paper, the analysis methods of displacement discontinuity in the process of material failure are discussed. It provides the theories foundation for exploring failure mechanism of displacement discontinuity, such as landslide, surface rupture of earthquake, etc.

\section{ACKNOWLEDGEMENT}

This work was supported by the National Natural Science Foundation of China under grant No.51508534, National Key Technology Support Program under grant No.2015BAK17B01, the National Natural Science Foundation of China under grant No. 51278474, Basic Science and Research Fund for Chinese Commonweal Institutes under grant No.2016A04, Basic Science and Research Fund for Chinese Commonweal Institutes under grant No.2014B06.

\section{REFERENCES}

[1] Washizu K., Variational methods in elasticity and plasticity, Cambridge University Press, 3rd edition, 1982.

[2] Rice J.R., The localization of plastic deformation, in: W.T. Koiter (Ed.), Theoretical and Applied Mechanics, North Holland Amsterdam, 1976, pp. 207-220.

[3] Bigon D., Bifurcation and Instability of Non-associative elastoplastic solids, CISM Courses and Lectures, 1-52. In Material instabilities and plastic solid, H. Petryk (ED.), Wien New York, Springer-Verlag 2000.

[4] Desrues J., Localisation Patterns in Ductile and Brittle Geomaterials, in Material Instabilities in Soils, Edited by Rene de Borst snd Erik, John Wiley \& Sons Ltd, 1998.

[5] Nicolas M., John D. and Ted Belytschko, A finite Element Method for Crack Growth without Remeshing, International Journal for Numerical Methods in Engineering. Vol.46, pp.131-150, 1999.

[6] Zienkiewicz O C, the Finite Element Method, McGraw Hill, London, 1971. 